\title{
Discerning Disorders and Diseases
}

\author{
By Jack M. Gorman, MD
}

Attention-deficit/hyperactivity disorder (ADHD) is just one of several psychiatric diagnoses to achieve notoriety in the mainstream press recently. With a ferociousness not generally applied to other medical conditions, psychiatric illness seems to be a favorite target of the media. Why do journalists feel comfortable opining on whether ADHD or childhood depression are "real" conditions, but never venture such opinions about asthma or gastritis?

One answer is that in the absence of objective evidence everyone feels entitled to have an opinion. Although asthma is an illness with less than perfect diagnostic margins and few directly diagnostic laboratory tests, the presence of wheezing on auscultation is a fairly certain sign that bronchospasm is the problem. Gastritis is diagnosed almost entirely on the basis of subjective complaint, although again the history of nausea, vomiting, and intense abdominal pain seems to add objective credence to the diagnosis. Psychiatric illness, however, overlaps with normal emotion. When does occasional distraction become ADHD, or moodiness merge into depression? Physical examination, laboratory testing, and radiograms offer no help to date in making these diagnoses and so, it seems, everyone feels qualified to venture an opinion about the validity of a psychiatric diagnosis.

For child psychiatrists who see cases of ADHD, the diagnosis is often not particularly subtle. A child who fidgets incessantly, interrupts, has temper outbursts, and does more poorly in school than intelligence testing predicts is a common patient seen by child psychiatrists. For them, the diagnosis is fairly straightforward. Moreover, their experience and hundreds of studies have shown that psychostimulants and certain other medications are clearly effective in improving the clinical status and quality of life of these children, as well as the well-being of their parents and teachers. I have seen pediatricians puzzle over whether a child has asthma just as often as I have seen child psychiatrists debate whether a child has $\mathrm{ADHD}$. The classic case is rarely problematic.

The issue for psychiatry remains, however, whether it is possible to locate basic neural processes that differentiate individuals with conditions like ADHD from those who do not have a diagnosis and do not need therapy. To get to this, one might ask how pediatricians know that the classic wheeze detected with a stethoscope actually represents a specific pathophysiology. The answer is that long before we knew exactly what asthma was, we knew how the lungs worked. We knew this in part from animal studies-the mammalian lung has not evolved all that much, so ours is fairly similar to those of many other animals. On the other hand, the ability to pay attention is clearly different in humans than in any other species.

Our ability to divide our attention among many competing inputs, to selectively suppress attention when necessary, and to focus on what is important are highly evolved cognitive processes. Attentional processes prominently involve the prefrontal cortex, particularly the anterior cingulate, an area of the brain that has gained considerable complexity and sophistication over evolutionary time.

Recently, Fan and colleagues ${ }^{2}$ showed that polymorphisms of two human genes, those coding for the enzyme monoamine oxidase $A$ and the dopamine 4 receptor, are linked to differences among normal individuals in the degree of anterior cingulate activation during an executive attention task, which in turn is linked to success at that task. This work is the beginning of understanding of how the brain accomplishes a particular task and how genes control differences in the general population in both brain response and success at performing that task. It is entirely analogous to our understanding of what is happening in the lung when a healthy person breathes and an asthmatic wheezes.

As we understand more about the basic neural circuitry and molecular genetics of a complex human cognitive activity like attention, it will become less difficult to understand what happens when attentional activity is abnormal. Then, one hopes, journalists will agree that psychiatric illnesses like ADHD are no more appropriate targets for casual dismissal than are asthma or gastritis. CNS

\section{REFERENCES}

1. Nimchinsky EA, Gilissen E, Allman JM, Perl DP, Erwin JM, Hof PR. A neuronal morphologic type unique to humans and great apes. Proc Natl Acad Sci U S A. 1999;96:5268-5273.

2. Fan J, Fossella J, Sommer T, Wu Y, Posner MI. Mapping the genetic variation of executive attention onto brain activity. Proc Natl Acad Sci U S A 2003;100:7406-7411 